\title{
Modulares IR-Messsystem zur Überwachung der Wasserstoffqualität für Brennstoffzellenfahrzeuge
}

\author{
M.Schott ${ }^{1}$, E.Pignanelli ${ }^{1}$, S.Gratz-Kelly ${ }^{2}$, A.Schütze S.,2 $^{1,2}$ \\ ${ }^{1}$ Zentrum für Mechatronik und Automatisierungstechnik gGmbH, 66121 Saarbrücken/GER \\ ${ }^{2}$ Universität des Saarlandes, Lehrstuhl für Messtechnik, 66123 Saarbrücken/GER \\ M.Schott@ZeMA.de
}

\begin{abstract}
Zusammenfassung
Geringe Verunreinigungskonzentrationen in Wasserstoff im ppm-Bereich, wie beispielsweise Kohlenmonoxid (CO) oder Kohlendioxid $\left(\mathrm{CO}_{2}\right)$, können in Brennstoffzellen leistungsreduzierende Effekte oder Schäden verursachen. Für Brennstoffzellen-betriebene Fahrzeuge, die eine vielversprechende Alternative zu konventionellen Verbrennungsmotoren sind, können derartige Verunreinigungen auch zum Liegenbleiben des Fahrzeugs führen. Um solche Störeinflüsse zuverlässig auszuschließen, muss die Reinheit von Wasserstoff überwacht werden. Die ersten Voruntersuchungen zeigen, dass ein Sensorsystem, das auf der nicht-dispersiven Infrarot (IR)-Absorption beruht, eine Vielzahl von Störgasen erfassen kann. Dabei wurde auch festgestellt, dass Wasserstoff bei hohem Druck IR-aktiv wird, die resultierende breite $\mathrm{H}_{2}$-Absorptionsbande überdeckt allerdings keine wesentlichen Verunreinigungsbereiche. Um höhere Gasdrücke in einem FTIR-Spektrometer untersuchen zu können, wurde ein Versuchsaufbau mit einem Druckverstärker konzipiert. Mit Hilfe des Lambert-BeerschenGesetzes und der Van-der-Waals-Gleichung wurde die zur Erzielung der gewünschten Empfindlichkeit erforderliche Länge des Messsystems abgeschätzt. Es wurde festgestellt, dass bei einem Druck von 700 bar, entsprechend dem Tankdruck von Brennstoffzellenfahrzeugen, viele relevante Gase wie Kohlenmonoxid oder Methan mit Küvettenlängen von einigen $10 \mathrm{~cm}$ gemessen werden können. Für die resultierende Druckverbreiterung und -verschiebung findet man in der Literatur verschiedene Modelle, die sich zwar für eine grobe Näherung eignen, aber für eine quantitative Aussage zu ungenau sind. Weitergehende experimentelle Untersuchungen sind daher erforderlich.
\end{abstract}

Keywords: Wasserstoff $\left(\mathrm{H}_{2}\right)$,Infrarot,Gasqualität,Hochdruck,Brennstoffzelle,IR-Aktivität.

\section{Motivation}

Wasserstoff eignet sich als kohlenstofffreier Energieträger hervorragend als Energiespeicher und Kraftstoff und gewinnt aufgrund der Energiewende immer mehr an Bedeutung. Wesentlicher Vorteil gegenüber Akkubetriebenen Elektrofahrzeugen ist die kurze Betankungszeit von maximal drei Minuten [1]. Daher genügt es, zentrale Betankungseinrichtungen wie bei konventionellen Benzin- und Dieselfahrzeugen $\mathrm{zu}$ errichten, statt viele dezentrale Ladestationen aufzubauen. So könnte man auf verteilte Einrichtungen, wie es aufgrund der langen Ladezeiten für die AkkuTechnologie unabdingbar ist, verzichten und erhebliche Kosten und Subventionen einsparen. Bereits jetzt wird intensiv in den Ausbau von Wasserstofftankstellen investiert. So werden bis $2018 / 19$ rund 100 Wasserstofftankstellen in sechs deutschen Ballungszentren und Verkehrsnetzen aufgebaut [2]. Geplant ist, dass in Deutschland bis zum Jahr 2023 bis zu 400 Wasserstofftankstellen zur Verfügung stehen [2]. Insgesamt wurden im Jahr 2016 so viele Wasserstofftankstellen eröffnet wie nie zuvor [3]. Weltweit waren es 92 Tankstellen, die ihre Pforten öffneten [3]. Besonders Dänemark stellt bereits jetzt ein flächendeckendes Netz und das beste Tankstelle-pro-PersonenVerhältnis bereit [3].

Bei Brennstoffzellen-betriebenen Fahrzeugen hat sich im PKW-Bereich aufgrund der geringen Energiedichte bei Umgebungsdruck die 700 bar Technologie durchgesetzt. Das bedeutet, dass der im Tank befindliche Wasserstoff auf einen Druck von 700 bar gebracht wird.

Für Fahrzeuge, die mit einer Brennstoffzelle versehen sind, spielt die Reinheit des eingebrachten Wasserstoffs eine zentrale Rolle, da sie effektiv die Lebensdauer der Brennstoffzelle beeinflusst und somit die Verfügbarkeit des Fahrzeuges entscheidend bestimmt. So kann beispielsweise Kohlen- 
monoxid eine katalytische Vergiftung [4] hervorrufen oder Membranschädigungen verursachen [5]. Für den Nachweis von Schadstoffen könnten prinzipiell kostengünstige Sensoren zum Einsatz kommen, z. B. elektrochemische Zellen oder Halbleitergassensoren. Ihr großer Nachteil liegt allerdings in der schlechten Selektivität und geringen Langzeitstabilität. Auch Gaschromatographen wären prinzipiell geeignet. Allerdings sind die hohen Kosten und die lange Messzeit nachteilig.

\section{Grundlagen und Messkonzept}

Bereits bei der online-fähigen Qualitätsüberwachung von Hydraulik- und Schmierölen konnte erfolgreich ein Messsystem realisiert werden, welches auf der nicht-dispersiven Infrarot (NDIR) Absorption beruht [6, 7, 8]. Darauf aufbauend soll dieser Ansatz auf die Qualitätsüberwachung von Wasserstoff übertragen werden. Der hohe Druck im $\mathrm{H}_{2}$-System erlaubt prinzipiell einen Nachweis von Schadstoffen auch im niedrigen ppm-Konzentrationsbereich. Allerdings ergeben sich aufgrund der im System vorherrschenden hohen Drücke neue Effekte, die vorab untersucht werden müssen. Der prinzipielle Aufbau des NDIRSensorsystems ist in Abbildung 1 dargestellt.

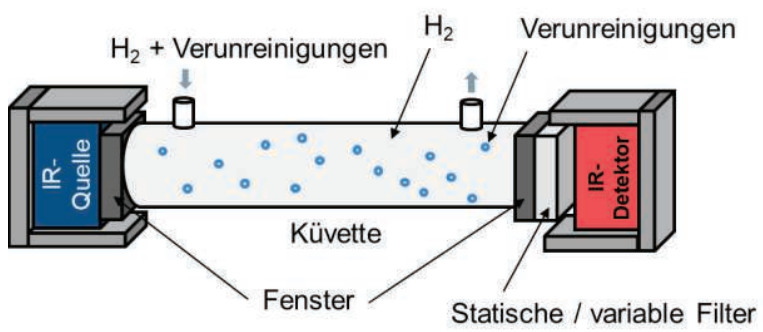

Abb. 1: Prinzipskizze des NDIR-Messsystems zur $\mathrm{H}_{2}$-Qualitätsüberwachung.

Das System besteht im Wesentlichen aus einer thermischen IR-Quelle (schwarzer Strahler), einem oder mehreren IR-Detektoren und gasspezifischen Filtern und einer Gasküvette. IR-Quelle und -Detektor werden von dem in der Küvette befindlichen Wasserstoff durch entsprechende IR-transparente Fenster abgetrennt. Diese müssen gleichzeitig den hohen Gasdrücken in der Küvette widerstehen. Die zwischenatomaren Bindungen der Gasmoleküle, in dem Fall Wasserstoff und Verunreinigungen, können durch die von der IR-Quelle ausgesandte elektromagnetische Strahlung zum Schwingen bzw. zu Rotationen angeregt werden. Entsprechend der Quantenmechanik ist die Aufnahme der Energien jedoch gequantelt, was dazu führt, das nur bestimmte Energieniveaus eingenommen und somit nur bestimmte Frequenzanteile aus der Strahlung absorbiert werden. Wird elektromagnetische Strahlung einer entsprechenden Frequenz durch das Medium gestrahlt, kann es mit den enthaltenen Molekülen wechselwirken, wodurch das Energieniveau des Moleküls erhöht und entsprechend die Strahlungsleistung verringert wird. Über die Stärke der Absorption kann somit auf die Konzentration zurückgeschlossen werden. Die für einzelne Moleküle charakteristischen Absorptionsspektren werden auch als Banden bezeichnet.

Nicht alle Molekülschwingungen sind IR-aktiv. Prinzipiell sind nur Moleküle IR-aktiv, welche entweder wie $\mathrm{CO}$ oder $\mathrm{H}_{2} \mathrm{O}$ ein permanentes Dipolmoment aufweisen und somit im elektromagnetischen Feld direkt zu Schwingungen angeregt werden, oder deren Dipolmoment sich bei der Schwingung ändert wie beim $\mathrm{CO}_{2}$, das kein permanentes Dipolmoment besitzt. Homonukleare Moleküle wie $\mathrm{H}_{2}$ oder $\mathrm{N}_{2}$ sind daher unter Normalbedingungen nicht IR-aktiv. Durch die thermische Bewegung der Moleküle kann jedoch bei Stoßvorgängen kurzzeitig ein Dipolmoment induziert werden, allerdings ist dies bei Normaldruck und -temperatur nicht ausreichend, um eine merkbare Schwingungsanregung und damit messbare IR-Absorption zu erzielen.

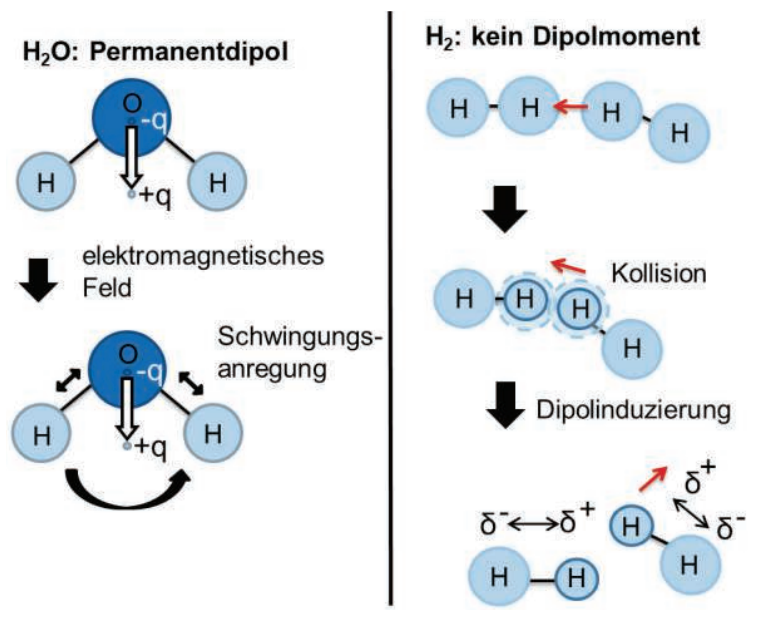

Abb. 2: Effekt der elektromagnetischen Strahlung auf ein unsymmetrisches Molekül und ein homonukleares Molekül bei erhöhtem Druck nach [9].

Bei hohen Drücken wird die Kollisionswahrscheinlichkeit der Moleküle jedoch deutlich erhöht, so dass in Wasserstoff vermehrt Dipolmomente induziert werden. Als Folge ist Wasserstoff bei hohem Druck IR-aktiv.

Entsprechend der Quantentheorie, nach der Moleküle nur durch elektromagnetische Strahlung einer ganz bestimmten Energie zum 
Schwingen angeregt werden können, besteht das Absorptionsspektrum aus einzelnen diskreten Linien (bzw. Peaks). Da sich neben der Schwingungsquantenzahl auch die Rotationsenergie um \pm 1 ändern muss, besteht das Absorptionsspektrum eines einzelnen Moleküls aus einer Vielzahl eng benachbarter Linien. In der Realität ergeben sich dabei keine unendlich schmalen Peaks, diese haben vielmehr eine gewisse Peakbreite. Die Peakbreite steigt bei hohem Druck weiter an, was als Druckverbreiterung $\Delta v_{c}$ bezeichnet wird. Ebenso stellt sich eine Druckverschiebung $\Delta \mathrm{v}_{0}$ ein. Beide Effekte sind in Abbildung 3 veranschaulicht.

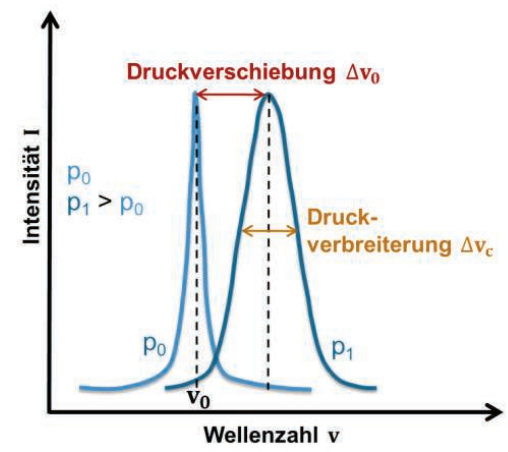

Abb. 3: Druckverbreiterung und Druckverschiebung bei erhöhtem Druck $p_{1}$ nach [10].

Anhand von theoretischen Modellen kann dieser Einfluss abgeschätzt werden. Hierzu wird der Kollisionskoeffizient für zwei Moleküle gemäß Gleichung (1) benötigt.

$$
\gamma_{i j}=\frac{r_{i j}}{c} \cdot \sqrt{\frac{16}{\pi \cdot \mu_{i j} \cdot k_{B} \cdot T}}
$$

Der Koeffizient $r_{i j}$ beschreibt den Streuradius und $\mu_{i j}$ die reduzierte Masse. Der resultierende Druckverbreiterungskoeffizient für das Molekül i lässt sich durch die einzelnen Molenbrüche $X_{j}$ und die Kollisionskoeffizienten sowie den vorherrschenden Druck $\mathrm{p}$ bestimmen.

$$
\Delta v_{c, i}=p \cdot \sum_{j} \gamma_{i j} X_{j}
$$

Ein weiterer wesentlicher Punkt ist die Küvettenlänge, um eine bestimmte Empfindlichkeit und damit die geforderte Nachweisgrenze zu erzielen. Je länger der Messweg ist, desto mehr Energie wird letztendlich von den Molekülen absorbiert. Die Abschätzung der Küvettenlänge für eine bestimmte Nachweisgrenze erfolgt in vereinfachter Form auf Basis des Lambert-Beersches-Gesetz nach Gleichung (3)

$$
L=\frac{-\log \left(\frac{I_{T}}{I_{0}}\right)}{\varepsilon(\lambda) \cdot c}
$$

IT beschreibt die Intensität des transmittierten Lichts, Io die Intensität des einfallenden Lichtes und $L$ die Küvettenlänge. Der dekadische Extinktionskoeffizient $\varepsilon(\lambda)$ wurde anhand der Datenbank ANSYCO berechnet [11]. c beschreibt die Konzentration des betrachteten Gases. Dabei wurden die Gasarten und Grenzwerte entsprechend der gültigen ISO-Norm [12] betrachtet. Bei Druckerhöhung steigt die absolute Gasmenge entlang des Absorptionswegs, was in Glg. (3) z. B. durch das ideale Gasgesetz berücksichtigt werden kann.

$$
p=\frac{R \cdot T}{V_{m}-b}-\frac{a}{V_{m}^{2}}
$$

$\mathrm{p}$ steht hierbei für den Druck, $V_{m}$ für das molare Volumen, $\mathrm{R}$ für die universelle Gaskonstante, $\mathrm{T}$ für die Temperatur, a für den Kohäsionsdruck und $b$ für das Kovolumen, d.h. die Konzentration steigt proportional zum Druck. Alternativ können auch andere Modelle, z. B. die ideale Gasgleichung, die Beziehung von Beattie-Bridgeman [13] oder Berthelot [14] verwendet werden. Je nach Ansatz ergeben sich geringfügig andere Weglängen für das Messsystem. In der Tabelle 1 sind für einige Verunreinigungen und für unterschiedliche Drücke die erforderliche Weglänge der Gasküvette unter der Annahme $I_{T} / l_{0}=0,995$ berechnet, d. h. es wurde ein Rauschniveau von $0,5 \%$ des Signals angenommen. Man erkennt, dass bei hohem Druck viele relevante Gase mit Küvettenlängen von einigen $10 \mathrm{~cm}$ gemessen werden können; von den in Tab. 1 gezeigten Gasen würde nur Formaldehyd $\left(\mathrm{CH}_{2} \mathrm{O}\right)$ deutlich längere Wege erfordern.

Tab. 1: Erforderliche Weglängen für verschiedene Verunreinigungen bei Drücken von 1, 10, 70 bzw. 700 bar

\begin{tabular}{|l|r|r|r|r|}
\hline \multirow{2}{*}{ Stoff } & \multicolumn{4}{|c|}{ Weglängen [m] } \\
\cline { 2 - 5 } & $p=1$ bar & $p=10$ bar & $b=70$ bar & $p=700$ bar \\
\hline $\mathrm{H}_{2} \mathrm{O}$ & 0,05 & 0,005 & 0,0005 & 0,0006 \\
\hline $\mathrm{CO}$ & 48,95 & 4,877 & 0,471 & 0,065 \\
\hline $\mathrm{CO}_{2}$ & 2,70 & 0,269 & 0,026 & 0,004 \\
\hline $\mathrm{NH}_{3}$ & 74,79 & 7,446 & 0,719 & 0,100 \\
\hline $\mathrm{CH}_{4}$ & 144,37 & 14,383 & 1,389 & 0,193 \\
\hline $\mathrm{CH}_{2} \mathrm{O}$ & 606,80 & 60,453 & 5,837 & 0,812 \\
\hline
\end{tabular}




\section{Messungen}

Um den Einfluss der Druckverschiebung sowie die IR-Aktivität des Wasserstoffs zu überprüfen, wurden erste Voruntersuchungen mit einer Hochdruckküvette durchgeführt.

Für die Messung wurde die Gasküvette zunächst mithilfe einer Gasmischanlage über Massenflussregler bei 1 bar mit Wasserstoff und $\mathrm{CO}_{2}$ befüllt. Anschließend wurde die Gasküvette über einen Hochdruckminderer mit reinem Wasserstoff befüllt und auf einen Druck von 65 bar gebracht; dabei wurde die $\mathrm{CO}_{2}$ Konzentration entsprechend reduziert. In Abbildung 4 ist eine schematische Skizze des Messaufbaus gezeigt.

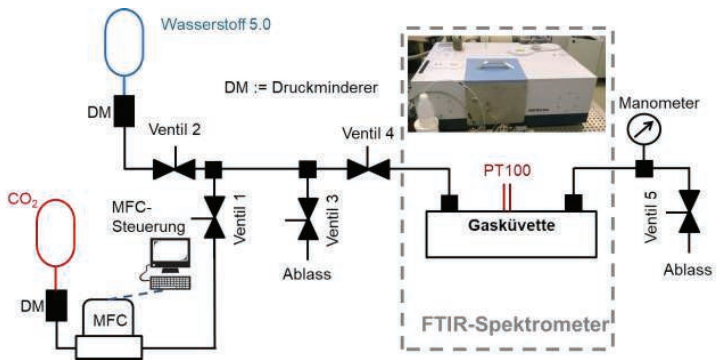

Abb. 4: Skizze des Versuchsaufbaus zur Untersuchung der Wasserstoff-IRAktivität bei Drücken bis 65 bar.

Zunächst wurde bei geöffnetem Ausgangsventil ein konstanter Massenstrom der beiden Eingangsleitungen eingestellt, um die Küvette zu spülen und die gewünschte Mischung aus $\mathrm{H}_{2}$ und $\mathrm{CO}_{2}$ einzustellen. Nachdem Eingangs- und Ausgangsventil geschlossen sind, wird die Wasserstoffflasche direkt über den Druckminderer an die Küvette angeschlossen, um diese auf einen entsprechend höheren Gesamtdruck zu bringen. Abschließend wurde die Hochdruck-Kurzweg-Küvette (Weglänge $4 \mathrm{~cm}$ ) in ein hochauflösendes FTIRSpektrometer (Bruker, Vertex 80v) eingebaut und mit diesem das Absorptionsspektrum bestimmt.

\section{Ergebnisse}

In den Abbildungen 5 und 6 sind die Absorptionsspektren für $3 \% \mathrm{CO}_{2}$ in reinem $\mathrm{H}_{2}$ bei Drücken von 1 bis 11,25 bar dargestellt; in den Abbildungen 7 und 8 für $0,5 \% \mathrm{CO}_{2}$ bei Drücken von 1 bis 65,25 bar. Alle Messungen wurden bei Raumtemperatur durchgeführt.

Die Details in Abb. 6 zeigen die $\mathrm{CO}_{2}$-Banden im Wellenzahlbereich von 3550 bis $3800 \mathrm{~cm}^{-1}$. Mit steigendem Druck sinkt - bedingt durch die größere Anzahl von Molekülen im Messweg die Transmission, d.h. die Empfindlichkeit des Messverfahrens steigt wie gewünscht an. Man erkennt bei niedrigen Drücken noch die einzelnen Linien des Spektrums, während diese bei höheren Drücken durch die Druckverbreiterung überlappen und eine breite Absorptionsbande bilden. Aus den Messungen lässt sich ein Druckverbreiterungskoeffizient von ca. $0,33 \mathrm{~cm}^{-1} /$ bar bestimmen, was unter dem nach der Theorie erwarteten Wert liegt.

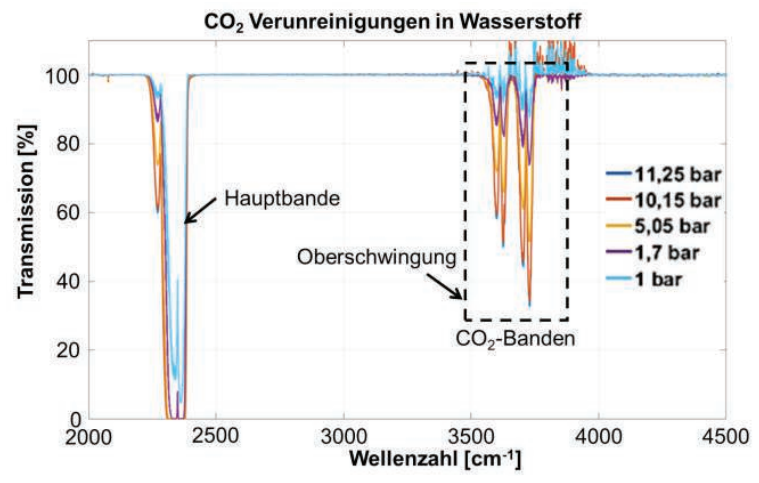

Abb. 5: IR-Absorptionsspektren bei Drücken von 1 bis 11,25 bar und $3 \% \mathrm{CO}_{2}$ nach [15].

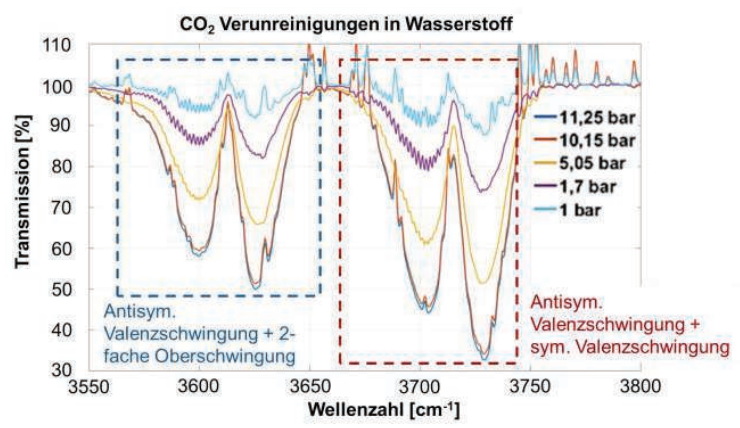

Abb. 6: Details der $\mathrm{CO}_{2}$-Banden im Wellenzahlbereich von 3550 bis $3800 \mathrm{~cm}^{-1}$ bei Drücken von 1 bis 11,25 bar und $3 \% \mathrm{CO}_{2}$ nach [15].

Von besonderem Interesse ist die Frage, ob und wenn ja in welchem Bereich und wie stark der Wasserstoff bei hohem Druck IR-aktiv wird. Dies kann bei einer 0,5\% $\mathrm{CO}_{2}$ Messung bei einem Druck von 65,25 bar beobachtet werden. In Abbildung 7 ist das Gesamtspektrum dargestellt, man erkennt die trotz des höheren Drucks geringere Abnahme der Transmission im Bereich der $\mathrm{CO}_{2}$-Banden aufgrund der geringeren Konzentration.

Abb. 8 zeigt, dass bei einem Druck von 65 bar zusätzlich zu den $\mathrm{CO}_{2}$ Banden zwischen ca. $3900 \mathrm{~cm}^{-1}$ und $5100 \mathrm{~cm}^{-1}$ eine weitere, breite Absorptionsbande erscheint, die auf die druckinduzierte IR-Aktivierung von Wasserstoff zurückzuführen ist. Bei ca. $5000 \mathrm{~cm}^{-1}$ ist zudem eine überlagerte $\mathrm{CO}_{2}$-Oberschwingung $\mathrm{zu}$ erkennen. Ein Vergleich mit simulierten Spektren (HITRAN-on-the-Web [16]) zeigt, 
dass sich hierbei größere Abweichungen im Hinblick auf die Druckverbreiterung ergeben. Besonders auffällig ist diese Abweichung wiederum bei der Messung bei 65 bar, wo die Druckverbreiterung der $\mathrm{CO}_{2}$-Hauptbande bei $2349 \mathrm{~cm}^{-1}$ in der Simulation deutlich größer ist als in der Messung. Die Abweichung kann zwar zum Teil durch die vereinfachte Konzentrationsberechnung (einfaches Druckverhältnis) bedingt sein, allerdings sollte dieser Fehler deutlich unter $5 \%$ liegen.

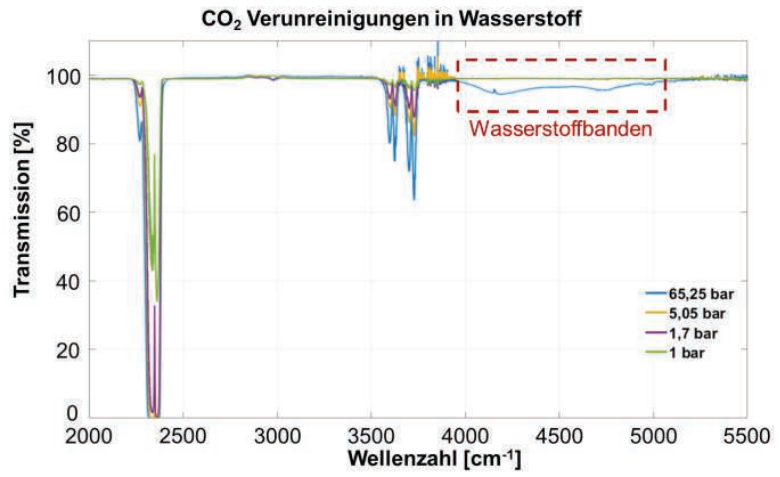

Abb. 7: IR-Absorptionsspektren bei Drücken von 1 bis 65,25 bar und 0,5\% CO nach [15].

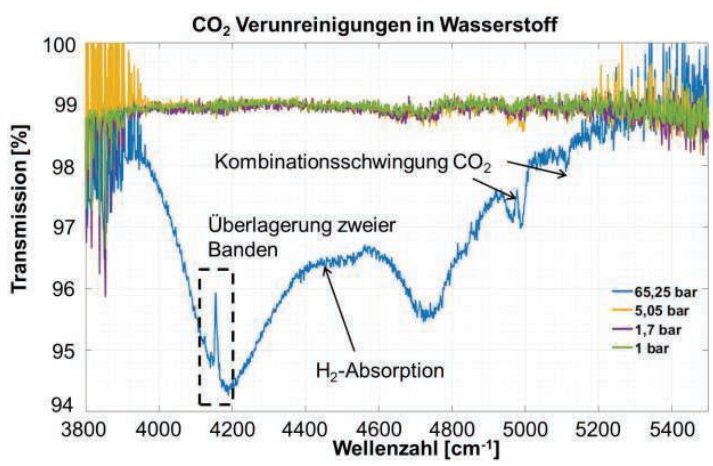

Abb. 8: Detail des IR-Spektrums im Wellenzahlbereich von 3800 bis $5500 \mathrm{~cm}^{-1}$ bei verschiedenen Drücken und 0,5\% $\mathrm{CO}_{2}$. Bei einem Druck von $65 \mathrm{bar}$ erscheint eine breite Absorptionsbande, die auf die druckinduzierte IR-Aktivierung von Wasserstoff zurückzuführen ist nach [15].

Die Voruntersuchungen zeigen also, dass die den Simulationen zugrundeliegenden Annahmen und Modelle offenbar für hohe Drücke nicht korrekt sind. Für die Auslegung des Messsystems ist daher eine umfassende experimentelle Charakterisierung bis zu dem später anvisierten Maximaldruck von 700 bar erforderlich. Die Voruntersuchungen liefern zudem die wesentliche Erkenntnis, dass die Wasserstoffbande bis 65 bar Druck keine wichtigen Absorptionsbanden der relevanten
Verunreinigungen überdeckt und dass die Druckverschiebung insgesamt offenbar vernachlässigt werden kann, da sie deutlich geringer ist als die Breite der Absorptionsbanden.

\section{Versuchskonzept für Drücke bis zu 925 bar}

Für Untersuchungen bei höheren Drücken wurde ein Versuchsaufbau konzipiert, der maximalen Drücken bis 925 bar standhält, siehe Abbildung 9. Wesentliche Änderung zu dem bereits vorhandenen Aufbau ist ein Druckverstärker, der den Gesamtdruck variabel erhöhen kann und einen maximalen Druck bis 925 bar erzielen kann. Für alle Versuche wird die Gasküvette wieder zunächst bei niedrigem Druck mit der gewünschten Verunreinigung in Wasserstoff gefüllt; anschließend wird das Gasgemisch mit reinem Wasserstoff verdünnt und auf den gewünschten Gesamtdruck gebracht. Bei den Messungen ist vorgesehen, zunächst den maximal gewünschten Druck einzustellen. Nach Durchführung einer FTIRMessung wird der Druck anschließend durch den kaskadierten Ventilaufbau um einen definierten Bruchteil reduziert. Dies erlaubt die Beobachtung der Absorptionsbanden bei variablen Drücken, um die Effekte von Druckverschiebung und -verbreiterung für verschiedene Verunreinigungen und Konzentrationen zu bestimmen und damit Kalibrierkennlinien für einzelne Verunreinigungen sowie für Gemische aufzunehmen.

Zusätzlich wird eine weitere Spülleitung direkt am Eingang des $\mathrm{H}_{2}$-Druckminderers eingebaut, mit der die Hauptleitung mit Argon gespült werden kann, um das gesamte System mit einem Inertgas zu reinigen. Stickstoff kommt hierfür nicht in Frage, da auch die Auswirkung von Stickstoff bei hohen Drücken gezielt untersucht werden soll.

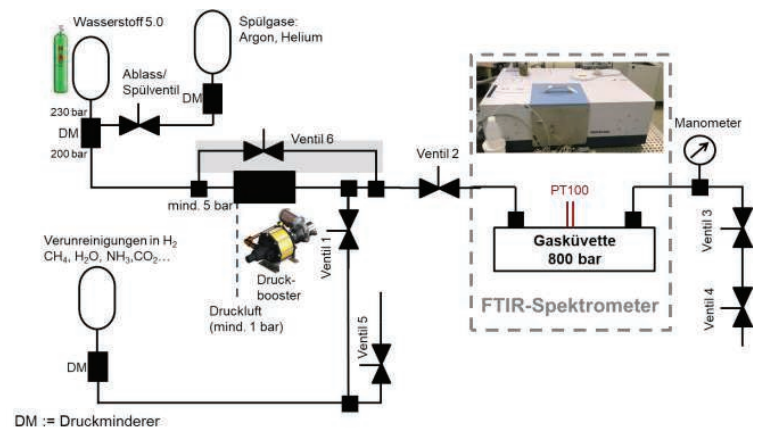

Abb. 9: Konzeptskizze des Versuchsaufbaus zur Untersuchung der Wasserstoffreinheit mittels IR-Absorption bei Drücken bis zu 925 bar. 


\section{Zusammenfassung}

Es konnte gezeigt werden, dass aufbauend auf vorherigen Ergebnissen ein Sensorsystem entwickelt werden kann, das die Reinheit von Wasserstoff zumindest für viele für Brennstoffzellen relevante Verunreinigungen online feststellen kann. Eine ausreichende Empfindlichkeit wird durch Messung bei hohem Druck, wie er beispielsweise in Brennstoffzellenfahrzeugen üblich ist, erzielt.

Allerdings absorbiert auch der normalerweise IR-inaktive Wasserstoff selbst bei hohen Drücken Strahlung in einem breiten Frequenzfenster von 3900 bis $5100 \mathrm{~cm}^{-1}$. Diese Überlagerung ist zumindest bis zu einem Druck von 65 bar unkritisch, da sie für die Messung der wesentlichen Verunreinigungen nicht störend ist. Ob dies auch noch für Drücke bis 700 bar gilt, muss in weiteren Untersuchungen gezeigt werden, da sich die Absorptionsspektren durch Druckverbreiterung und -verschiebung verändern und auch ihre relative Lage sich ändern kann. Für Untersuchungen bei sehr hohem Druck bis 925 bar wurde ein neuer Versuchsaufbau konzipiert, mit dem dieser Sachverhalt systematisch untersucht werden kann. Theoretische Modelle für Druckverbreiterung und -verschiebung können zwar als erste Näherung verwendet werden, sie ersetzen allerdings die experimentelle Untersuchung nicht, da die bestehenden Modelle offenbar bei hohem Druck nicht ausreichend genau sind.

\section{Danksagung}

Die zugrunde liegenden Arbeiten wurden vom Bundesministerium für Wirtschaft und Energie (BMWi) über den Projektträger PTJ im Rahmen des Projekts HAIMa $\left(\mathrm{H}_{2}-\right.$ und KationenKontamination: Alterungseffekte, Material- und Sensorentwicklung; Teilvorhaben: Entwicklung und Erprobung von Messprinzipien zur $\mathrm{H}_{2-}$ Qualitätsüberwachung) gefördert.

\section{Literaturnachweis}

[1] http://www.shell.de/energie-undinnovation/mobilitaet/wasserstoff.html, Stand 23.10.2017.

[2] http://www.nucellsys.com/produkt-technologie, Stand: 24.10.2017.

[3] 9. Jahresauswertung von LBST und TÜV SÜD, http://www.lbst.de/.

[4] R. Peters, Brennstoffzellensysteme in der Luftfahrt, Springer Vieweg, 2015.

[5] K. Borgeest, Elektronik in der Fahrzeugtechnik, Hardware, Software, Systeme und Projektmanagement, 2. Auflage, Vieweg Teubner, 2010.
[6] T. Bley, A. Schütze: A Multichannel IR Sensor System for Condition Monitoring of Technical Fluids, Proceedings IRS2 2011 - 12th International Conference on Infrared Sensors and Systems, Nürnberg, June 7 - 8, 2011; doi: 10.5162/irs11/i4.3.

[7] T. Bley, E. Pignanelli, A. Schütze: COPS Combined Oil quality and Particle measurement System, Proceedings ICST 2012 - The Sixth International Conference on Sensing Technology, Kolkata, India, December 18-21, 2012; doi:

10.1109/ICSensT.2012.6461742

[8] E. Pignanelli, T. Bley, A. Schütze: Modulares Condition Monitoring System zur Überwachung von Fluidqualität und Partikelkontamination am Beispiel von Öl, 11. Dresdner Sensor Symposium, Dresden, 9.-11. Dezember 2013; doi 10.5162/11dss2013/E8

[9] D. A. Mayer, A Fourier Transform - Infrared Spectroscopy Study of Hydrogen Interaction with Metal-Organic-Frameworks, Masterthesis, University of New Jersey, 2007.

[10] A. T. Brysch, Laser-Emissionsspektrometrie für die Partikelanalyse von Prozessgasen bei der Roheisenerzeugung, Dissertation, Rheinisch-Westfälische Technische Hochschule Aachen, Fakultät für Maschinenwesen, 2004.

[11] http://www.ansyco.de/

[12] SAE J 2719: Hydrogen Fuel Quality for Fuel Cell Vehicles.

[13] R.M. Dreizler, C.S. Lüdde, Theoretische Physik 4, Statistische Mechanik und Thermodynamik, Springer Verlag, 2016.

[14] T. Engel, P. Reid, Physikalische Chemie, Pearson Studium, 2006.

[15] S. Gratz-Kelly, Untersuchungen zur Detektion von Verunreinigungen in Wasserstoff bei hohen Drücken mittels Infrarot-Spektroskopie, Masterarbeit, Universität des Saarlandes, 2017.

[16] http://hitran.iao.ru/bands/simlaunch?mol=5 